\title{
Comparison of human amniotic fluid-derived and umbilical cord Wharton's Jelly-derived mesenchymal stromal cells: Characterization and myocardial differentiation capacity
}

\author{
Jing $\mathrm{Bai}^{1 *}$, Yuan $\mathrm{Hu}^{2}$, Yi-Ru Wang ${ }^{3}$, Li-Feng $\mathrm{Liu}^{3}$, Jie Chen ${ }^{3}$, Shao-Ping Su${ }^{4}, \mathrm{Yu} \mathrm{Wang}{ }^{3}$ \\ ${ }^{1}$ Department of Cardiology, The First Affiliated Hospital of Chinese PLA General Hospital, 51 Fucheng Road, Haidian District, Beijing 100048 , China \\ ${ }^{2}$ Institute of Orthopedics, The First Affiliated Hospital of Chinese PLA General Hospital, 51 Fucheng Road, Haidian District, Beijing 100048, China \\ ${ }^{3}$ Institute of Geriatric Cardiology, Chinese PLA General Hospital, 28 Fuxing Road, Haidian District, Beijing 100853, China \\ ${ }^{4}$ The $9^{\text {th }}$ Consulting Room, Chinese PLA General Hospital, 28 Fuxing Road, Haidian District, Beijing 100853, China
}

\begin{abstract}
Objective To compare the characterization and myocardial differentiation capacity of amniotic fluid-derived mesenchymal stromal cells (AF MSCs) and umbilical cord Wharton's Jelly-derived mesenchymal stromal cells (WJ MSCs). Methods The human AF MSCs were cultured from amniotic fluid samples obtained by amniocentesis. The umbilical cord WJ MSCs were obtained from Wharton's Jelly of umbilical cords of infants delivered full-term by normal labor. The morphology, growth curves, and analyses by flow cytometry of cell surface markers were compared between the two types of cells. Myocardial genes (GATA-4, c-TnT, $\alpha$-actin, and Cx43) were detected by real-time PCR and the corresponding protein expressions were detected by Western blot analysis after myocardial induced in AF MSCs and WJ MSCs. Results Our findings revealed AF MSCs and WJ MSCs shared similar morphological characteristics of the fibroblastoid shape. The AF MSCs were easily obtained than the WJ MSCs and had a shorter time to reach adherence of $2.7 \pm 1.6$ days to WJ MSCs of $6.5 \pm 1.8$ days. The growth curves by MTT cytotoxic assay showed the AF MSCs had a similar proliferative capacity at passage 5 and passage 10 . However, the proliferative capacities of WJ MSCs were decreased at 5 passage relative to 10 passage. Both AF stem cells and WJ stem cells had the characteristics of mesenchymal stromal cells with some characteristics of embryonic stem cells. They express CD29 and CD105, but not CD34. They were positive for Class I major histocompatibility (MHC I) antigens (HLA-ABC), and were negative, or mildly positive, for MHC Class II (HLA-DR) antigen. Oct-4 was positive in all the two cells types. Both AF MSCs and WJ MSCs could differentiate along myocardium. The differentiation capacities were detected by the expression of GATA-4, c-TnT, $\alpha$-actin, Cx43 after myocardial induction. Conclusions Both AF MSCs and WJ MSCs have the potential clinical application for myogenesis in cardiac regenerative therapy.
\end{abstract}

J Geriatr Cardiol 2012; 9: 166-171. doi: 10.3724/SP.J.1263.2011.12091

Keywords: Human amniotic fluid-derived cells; Wharton's Jelly-derived cells; Myocardial differentiation; Mesenchymal stromal cells

\section{Introduction}

It is widely agreed that cellular cardiomyoplasty has the potential to be an ideal method for the treatment of heart failure, which is a major cause of mortality and morbidity worldwide. $^{[1]}$ Stem cells from many sources have been explored for their advantages and limitations in clinical use. After a decade of stem cell studies, there were still no consensus conclusions on bone marrow mesenchymal stromal cells use

*The first two authors contributed equally to this paper

Correspondence to: $\mathrm{Yu}$ Wang, $\mathrm{PhD}$, Institute of Geriatric Cardiology, Chinese PLA General Hospital, 28 Fuxing Road, Haidian District, Beijing 100853, China. E-mail: wangyuheart@yahoo.com.cn

Telephone: $+86-13701246810 \quad$ Fax: $+86-010-55499037$

Received: December 9, $2011 \quad$ Revised: April 6, 2012

Accepted: April 13, 2012

Published online: May 28, 2012 in heart muscle cell regeneration, for adult stem cells have poorly characterized growth conditions and cannot be propagated quickly in vitro. Thus, it is not suitable for optimal transplantation time which has been confirmed in several clinical trials of four to seven days for acute myocardial infarction. Therefore, allogeneic transplantation methods need to be developed to address the issue of narrow transplantation time window in acute myocardial infarction. Amniotic fluid and umbilical cords are newly found sources containing mesenchymal stromal cells, and is a promising alternative source for cellular therapy. Current studies have shown some advantages of the AF MSCs and WJ MSCs for their strong proliferation capacity in vitro and their multiple differentiation capacity along all three germs. ${ }^{[2-4]}$ However, the mesenchymal stromal cells from different tissue sources may have different bio-characteristics and myocardial differentiation capacity. Our main objectives were to better charac-

http://www.jgc301.com; jgc@jgc301.com | Journal of Geriatric Cardiology 
terize the AF MSCs and WJ-MSCs and compare the myocardial differentiation potential of the two cell types.

\section{Methods}

\subsection{Samples}

We obtained 12 human amniotic fluid samples by amniocentesis performed between 18 and 28 weeks of gestation for prenatal diagnosis and legal terminations. This is an ethically approved and accepted procedure in the First Affiliated Hospital of the Chinese PLA General Hospital. All patients have signed informed consents. Cell samples were only used when no major abnormalities were revealed by the cytogenetic analysis. Nine human umbilical cord samples were aseptically collected from delivered, full-term infants by normal labor. The written, informed consents were obtained from the family members of the newborns and the experimental protocol was approved by the Ethics Committee of the First Affiliated Hospital of Chinese PLA general Hospital Ethics Committee.

\subsection{Isolation, culture and passaging of AF MSCs and WJ MSCs}

Cells were isolated from the AF samples no more than four hours prior to being used. The samples were centrifuged at $1500 \mathrm{r} / \mathrm{min}$ for 5 minitues. After removal of supernatant, the precipitation was cultured at $37^{\circ} \mathrm{C}$ in a humidified atomsphere containing $5 \% \mathrm{CO}_{2}$ after routine manual cells counting. The AF cells culture medium was composed of Minimum Essential Medium ( $\alpha$-MEM; Gibco, Langley, OK, USA) supplemented with 20\% Chang Medium (18\% Chang B plus $2 \%$ Chang C; Irvine Scientific, Santa Ana, CA, USA), 20\% Embryonic Stem Fetal Bovine Serum (ES FBS; Gibco, Langley, OK, USA), 1\% glutamine and 1\% penicillin/ streptomycin. After three days, non-adherent cells and debris were discarded and the adherent cells were continued to be cultivated in pre-confluency. The Morphologic characteristics of the AF cells were observed with inverted phase contrast microscope (Olympus IX51; Tokyo, Japan). Cells were dissociated by trypsin-EDTA solution (Invitrogen, San Francisco, CA, USA), when they reached $80 \%-90 \%$ confluence. To isolate WJ MSCs from Wharton's Jelly, 4-5 cm segments of umbilical cord were obtained, and the umbilical vein and two arteries were removed. Wharton's Jelly was processed within 24 hours of collection and cut into pieces of about $1 \mathrm{~mm}^{3}$ for culture. These pieces were placed in culture plate and cultured in DMEM supplemented with $10 \%$ fetal bovine serum, $5 \mathrm{ng} / \mathrm{mL}$ epidermal growth factor, $5 \mathrm{ng} / \mathrm{mL}$ basic fibroblast growth factor, $100 \mathrm{u} / \mathrm{mL}$ penicillin and $100 \mathrm{mg} / \mathrm{mL}$ streptomycin, and $1 \mu \mathrm{g} / \mathrm{mL}$ amphoterin $\mathrm{B}$. The culture plate was placed in an incubator with saturated humidity at $37^{\circ} \mathrm{C}$ containing $5 \% \mathrm{CO}_{2}(\mathrm{v} / \mathrm{v})$. The medium was changed every three days and the cells were passaged when reaching $80 \%$ confluence. Adherent cells were removed by treatment with $0.25 \%$ trypsin for 3 to 5 minitues then centrifuged.

\subsection{Growth curves}

The AF MSCs and WJ MSCs of passage 5 and 10 were respectively trypsinized and inoculated in 96-well culture plates to a total volume of $100 \mu \mathrm{L} /$ well (3000 cells/well) and incubated at $37^{\circ} \mathrm{C}$ in a humidified atmosphere containing $5 \%$ $\mathrm{CO}_{2}$. These cells were allowed to attach overnight and then incubated for seven days. Control wells for absorbance readings contained no cells except for the culture medium. Cell viability was determined by MTT cytotoxic assay. Triplicate wells were supplemented with $20 \mu \mathrm{L}$ MTT solution every day. After four hours of incubation, the absorbance was measured at $490 \mathrm{~nm}$ using an enzyme micro-plate reader (Bio-Rad Laboratories, Berkeley, CA, USA).

\subsection{Flow cytometry}

Cell surface markers on MSCs were analyzed using a panel of antigens of AF cells and WJ cells at passage 5- 6 by flow cytometric analysis. Cells in culture were trypsinized, washed and resuspended in phosphate-buffered saline (PBS; Sigma-Aldrich; Louis, MO, USA) at concentration of $1 \times$ $10^{5}$ cells $/ 100 \mu \mathrm{L}$. Cells were stained directly with FITC-immunolabeled mouse anti-human monoclonal antibodies against CD29, CD105, CD34, HLA-ABC, HLA-DR (Becton Dickinson, Berkeley, CA, USA) and rabbit anti-human antibodies against Oct-4 (Becton Dickinson, Berkeley, CA, USA). Analysis was performed with flow cytometer (Cytomics FC 500 MPL; Bechman Coulter, Brea, CA, USA) and data were analyzed with the Cxp Software. Data were expressed as number of cells $/ 10^{6}$ cytometric events.

\subsection{Myocardium-like cells differentiation}

The AF MSCs and WJ MSCs were respectively seeded at a density of 3000 cells $/ \mathrm{cm}^{2}$ on plastic plates precoated with Matrigel (Collaborative Biomedical Products; incubation for one hour at $37^{\circ} \mathrm{C}$ at $1 \mathrm{mg} / \mathrm{mL}$, in DMEM) in DMEM lowglucose formulation containing 10\% horse serum (Gibco/ BRL), $0.5 \%$ chick embryo extract (Gibco/BRL) and Pen/ Strep. Twelve hours after seeding, $3 \mu \mathrm{mol} / \mathrm{L}$ 5-aza-2'-deoxycytidine (5-aza-C; Sigma-Aldrich) were added to the culture medium for 24 hours. Incubation then continued in complete medium lacking 5-azaC, with medium changes every three days. Undifferentiated cells were used as controls. 


\subsection{Real-time Polymerase Chain Reaction (real-time PCR)}

The total mRNA was extracted with Trizol Reagent (Invitrogen, San Francisco, CA, USA) from the AF MSCs and WJ MSCs. Transcriptional expressions of GATA-4, c-TnT, $\alpha$-actin, and Cx43 genes were determined by real time PCR according to the manufacture's instructions. Transcript levels were standardized to the corresponding human GAPDH level. The primers for Realtime PCR and products size were shown in Table 1.

Table 1. The primers for Realtime PCR and products size.

\begin{tabular}{llc}
\hline Gene ( marker ) & Primer sequence & Size (bp) \\
\hline GATA-4 & F5'-CTGTGCCAACTGCCAGACCA-3' & 437 \\
& R5'-GGCTGACCGAAGATGCGTAG-3' & \\
c-TnT & F5'-GGCAGCGGAAGAGGATGCTGAA-3' & 150 \\
& R5'-GAGGCACCAAGTTGGGCATGAACGA-3' & \\
\multirow{2}{*}{$\alpha$-actin } & F5'-ATCTATGAGGGCTACGC-3' & 119 \\
& R5'-GCAGTGGTGACAAAGGA-3' & \\
Cx43 & F5'-GAATCCTGCTCCTGG-3' & 380 \\
& R5'-GATGCTGATGATGTAG-3' & \\
GAPDH & F5'-GCTTGTCATCAATGGAAATCCC-3' & 360 \\
& R5'-TCCACACCCATGACGAACATG-3' & \\
\hline
\end{tabular}

\subsection{Western blot analysis}

For western blot analyses, protein extracts were prepared in buffer containing $20 \mathrm{mmol} / \mathrm{L}$ HEPES ( $\mathrm{pH} 7.9$ ), $0.4 \mathrm{~mol} / \mathrm{L}$ $\mathrm{NaCl}, 2.5 \%$ glycerol, $1 \mathrm{mmol} / \mathrm{L}$ EDTA, $1 \mathrm{mmol} / \mathrm{L}$ phenylmethylsulfonyl fluoride, $0.5 \mathrm{mmol} / \mathrm{L} \mathrm{NaF}, 0.5 \mathrm{mmol} / 1 \mathrm{Na}_{3} \mathrm{VO}_{4}$, $0.02 \mu \mathrm{g} / \mathrm{mL}$ leupeptin, $0.02 \mu \mathrm{g} / \mathrm{mL}$ aprotinin, $0.003 \mu \mathrm{g} / \mathrm{mL}$ benzamidne chloride, $0.1 \mu \mathrm{g} / \mathrm{mL}$ trypsin inhibitor and $0.5 \mathrm{mmol} / \mathrm{L}$ dithiothreitol (DTT). Proteins were resolved on denaturing SDS-polyacrylamide gels (10\%), transferred to Immuno-Blot poly-vinylidene difluoride membranes (Bio-Rad Laboratories, Berkeley, CA, USA). Western blots were probed with goat anti-human primary antibodies (anti-GATA-4, anti-c-TnT, anti- $\alpha$-actin, and anti-Cx43). Primary antibody bindings were detected with horseradish peroxidase-conjugated rabbit antigoat (Zymed Laboratories, South San Francisco, CA, USA) and visualized using the ECL plus Western Blotting Detection System (Bio-Rad Laboratories).

\subsection{Statistical analysis}

Statistical analysis was performed with the SPSS 10.0 statistics software. Data were analyzed for statistical significance by Student's $t$ test. Data are presented as mean $\pm \mathrm{SD}$ for statistical analysis. A value of $P<0.05$ was considered to reveal significant statistical difference.

\section{Results}

\subsection{Morphology and growth curves}

AF samples from 18 weeks to 28 weeks of gestation were analyzed. The newly obtained AF cells were morphologically heterogeneous and were suspended in the culture medium (Figure 1A). AF cells were allowed to adhere to culture dishes and were grown for passaging in AF cells medium. The newly obtained WJ MSCs were morphologically similar and with more tissue debris in culture medium (Figure 1B). The average time to reach adherence was $2.7 \pm$ 1.6 days of AF MSCs and $6.5 \pm 1.8$ days of WJ MSCs. After passaging, the AF MSCs and the WJ MSCs grew conglomerately with homogeneous fibroblastoid shapes (Figure $1 \mathrm{C} \& \mathrm{D}$ ). MTT proliferation analysis showed that the AF MSCs and the WJ MSCs from passage 5 and passage 10 had similar growth curves (Figure 2).
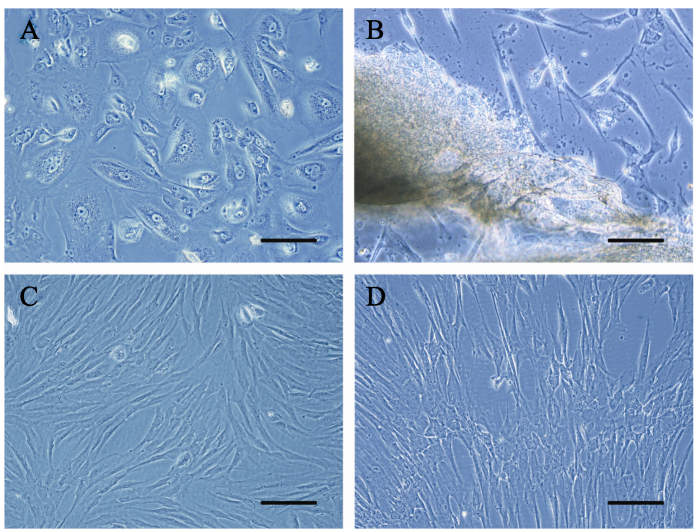

Figure 1. Morphological characteristics of AF MSCs and WJ MSCs. (A): Cultured primary amniotic fluid cells were a heterogeneous population $(\times 200)$; $(\mathrm{B})$ : Primary cultured umbilical cord WJ MSCs with tissue debris $(\times 200)$; $(\mathrm{C})$ : After passaging, the AF MSCs were fibroblastoid shapes $(\times 200)$; (D): The WJ MSCs grew conglomerately with a homogeneous fibroblastoid shapes $(\times 200)$. The bars in all pictures are $100 \mu \mathrm{m}$. AF MSC: amniotic fluidderived mesenchymal stromal cells, WJ MSCs: Wharton's Jellyderived mesenchymal stromal cells.

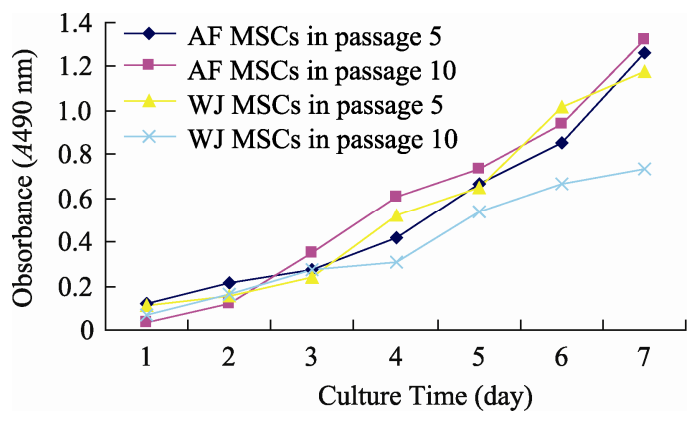

Figure 2. Growth curves of AF MSCs and WJ MSCs of Passage 5 and Passage 10. The growth curves of AF MSCs in passage 5, 10 and WJ MSCs in passage 5 were similar. The growth curves of WJ MSCs in passage 10 showed relatively decreased propagation rate. Results were shown from three independent experiments. AF MSC: amniotic fluid-derived mesenchymal stromal cells; WJ MSCs: Wharton's Jelly-derived mesenchymal stromal cells. 
3.2 Flow cytometry analysis of AF MSCs and WJMSCs

The characteristics of cell surface antigens of AF MSCs and WJ MSCs at passage 5-6 were analyzed by flow cytometry. Both AF MSCs and WJ MSCs were positive for mesenchymal stromal cells surface markers including CD29, CD105, and were negative for hematopoietic markers CD34. Both AF MSCs and WJ MSCs were positive for Class I major histocompatibility (MHC I) antigens (HLA-ABC), and were negative, or mildly positive, for MHC Class II (HLA-
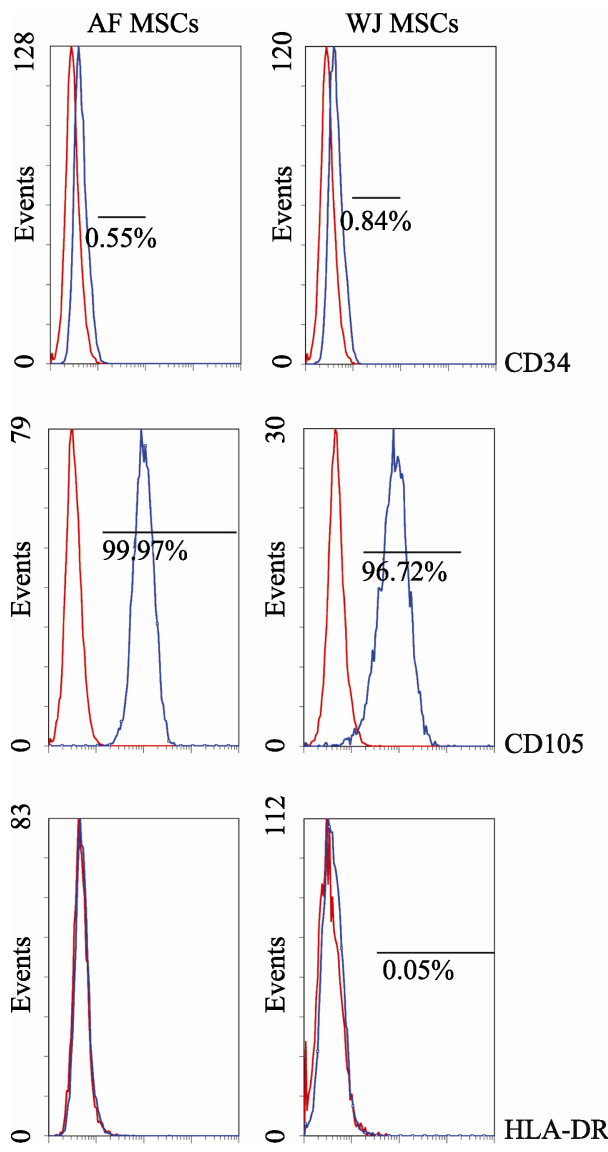

DR) antigen. Oct-4 was detected from AF MSCs and WJ MSCs (Figure 3).

\subsection{Myocardium-like cells differentiation}

Following myocardial differentiation, real-time PCR showed that myocardial genes GATA-4, c-TnT, $\alpha$-actin, and Cx43 were both detected in AFSCs and WJ-MSCs differentiated cells (Figure 4). Western blot showed that myocardial genes GATA-4, c-TnT, $\alpha$-actin, and Cx43 were detected in induced AF MSCs and WJ MSCs (Figure 5).
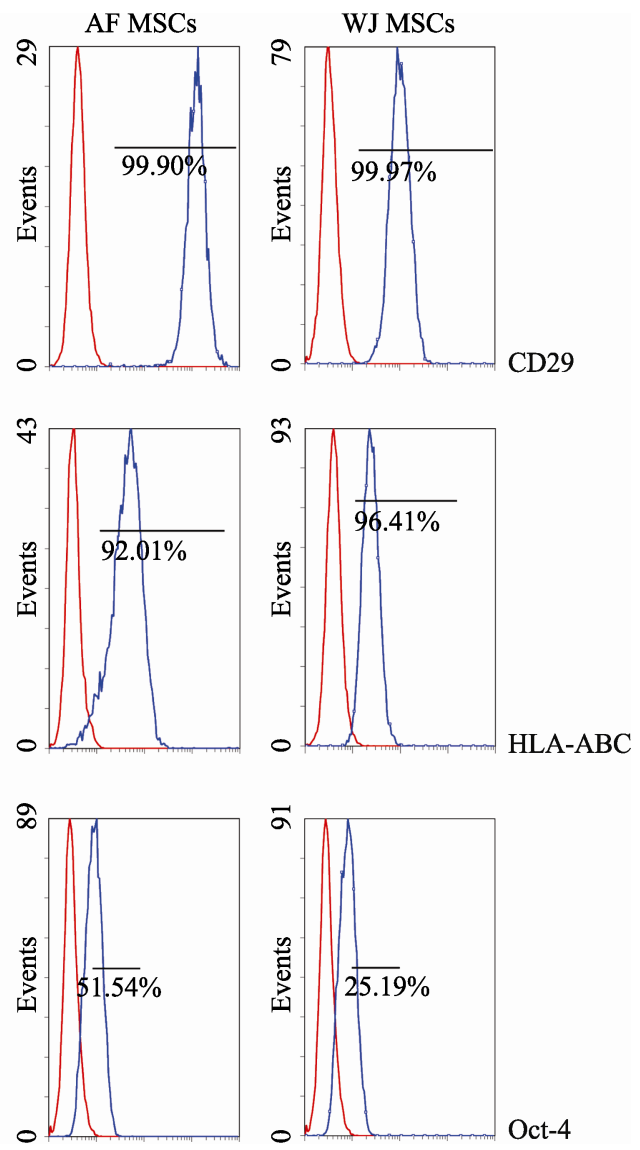

Figure 3. Flow cytometry analyses of AF MSCs and WJ MSCs. Red line corresponds to the isotype IgG control and the blue line to the antibody of interest. Results were shown from three independent experiments. AF MSC: amniotic fluid-derived mesenchymal stromal cells, WJ MSCs: Wharton's Jelly-derived mesenchymal stromal cells.

\section{Discussion}

MSCs were initially isolated from bone marrow by Friedenstein et al. ${ }^{[5]}$ MSCs have been found to have the potential to differentiated into muscle cells, adipocytes, osteocytes and chondrocytes in culture. ${ }^{[6-8]}$ The umbilical cord contains two arteries and one vein, which are surrounded by mucoid connective tissue, and this is called Wharton' Jelly. Human amniotic fluid and Umbilical cord Wharton's Jelly are plentiful and new sources of mesenchymal stromal cells were newly found. ${ }^{[9-12]}$ Several studies have investigated that the AF MSCs and WJ MSCs show either meshenchymal stem cell characteristics, or embryonic stem cell characteristics. They express the mesenchymal stromal cell surface markers, such as CD29, CD44, CD73, CD90 and CD105, and also the embryonic stem cell markers, such as Oct-4, SSEA-4 and Naong, etc. ${ }^{[13]}$ Thus, they were considered to be the intermediate stage of adult stem cells and embryonic stem cells. These cells may possess more advantages than adult 

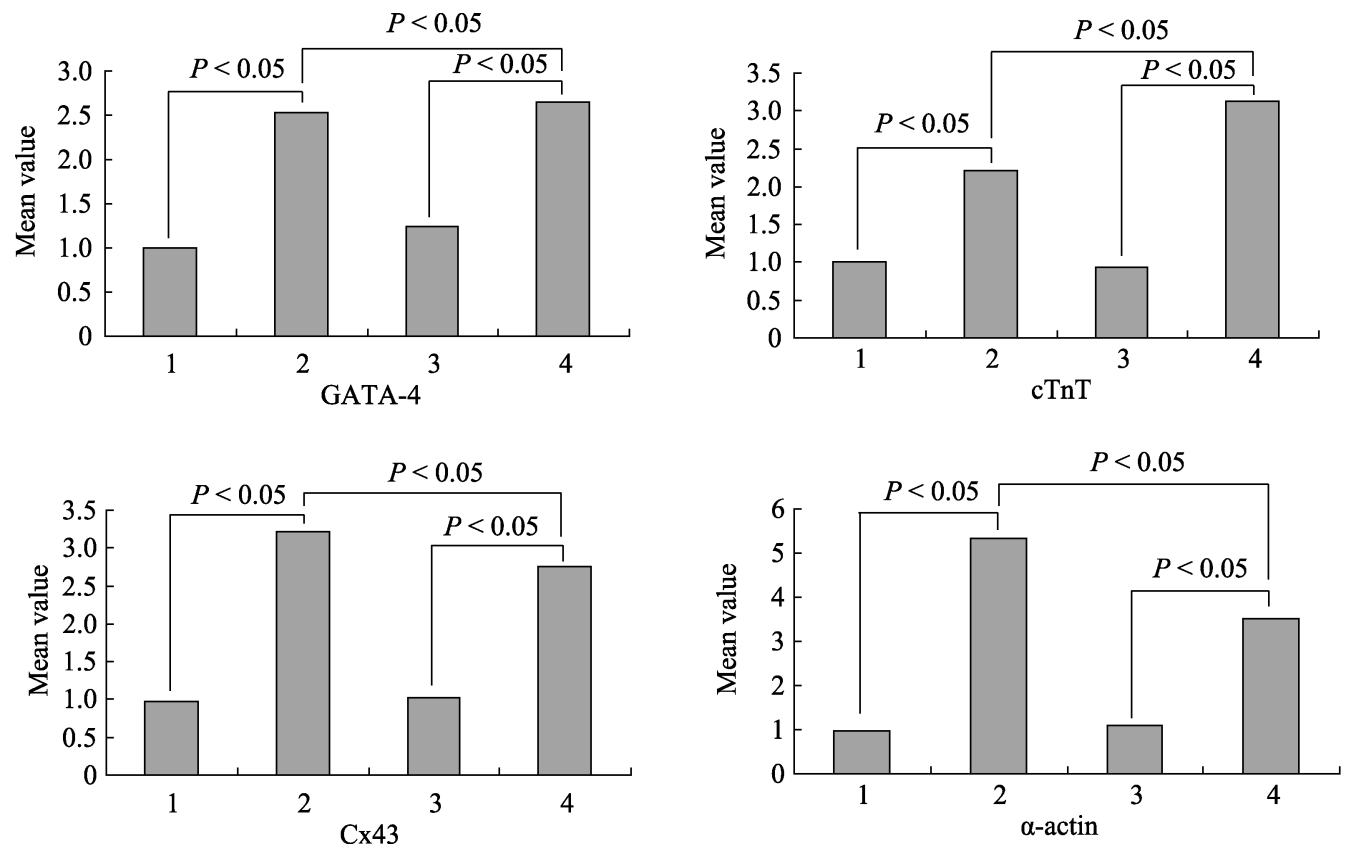

Figure 4. Real-time PCR analyses of GATA-4, c-TnT, $\boldsymbol{\alpha}$-actin and Cx43 genes. 1: Undifferentiated AF MSCs; 2: Myocardial induced AF MSCs; 3: Undifferentiated WJ MSCs; 4: Myocardial induced WJ MSCs. Results were shown as means \pm SD from three independent experiments. For real-time PCR, transcript levels were standardized to the corresponding human GAPDH level. AF MSC: amniotic fluid-derived mesenchymal stromal cells, WJ MSCs: Wharton's Jelly-derived mesenchymal stromal cells.

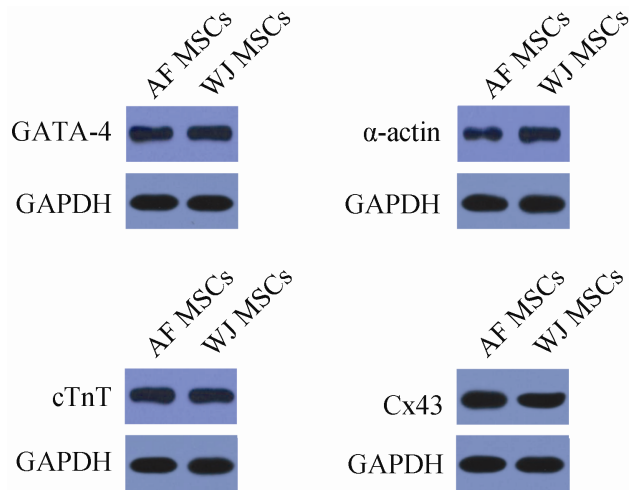

Figure 5. Western blot analysis of GATA-4, c-TnT, $\alpha$-actin and $\mathrm{Cx} 43$ protein expression were performed in AF MSCs and WJ MSCs. Results were shown from three independent experiments. AF MSC: amniotic fluid-derived mesenchymal stromal cells, WJ MSCs: Wharton's Jelly-derived mesenchymal stromal cells.

stem cells, such as stronger proliferation capacity in vitro and multipotent differentiation capacity. Our findings revealed that the average time to reach adherence was $2.7 \pm 1.6$ days for AF MSCs and $6.5 \pm 1.8$ days for WJ MSCs. The significant different may be due to the different harvesting methods. AF MSCs can be directly centrifuged from amniotic fluid, but the WJ MSCs must be isolated from tissue by cutting tissues or trypsinization. The primary cultured amniotic fluid stem cells were suspended in the culture medium with fibroblast-like, epithelial-like or irregularity morphology.
The primary cultured umbilical cord WJ MSCs were a homogenous cell population. After passaging, the AF MSCs and WJ MSCs exhibited fibroblast-like appearance, which were in line with a previous report. ${ }^{[14]}$ These two types of cells could proliferate for more than 50 passages in our culture study. The growth curves were similar in passage 5 of both two cell types. However, we can see different growth curves in passage 10 of a relatively decreased propagation rate in $\mathrm{WJ}$ MSCs. In our study, both the two cell types showed mesenchymal stromal cell phenotypes. The cultured cells were strongly positive for mesenchymal stromal cell markers (CD29, CD105), negative for hematopoietic marker CD34, positive for Class I MHC antigens (HLA-ABC) and negative, or mildly positive, for MHC Class II antigen (HLA- DR). By flow cytometric analyses, Oct- 4 was positive in $51.54 \%$ of AF MSC cells and $25.19 \%$ of WJ MSC. Oct- 4 had been reported as associated with the maintenance of the undifferentiated state and the pluri-potency of ES and EG cells. ${ }^{[9]}$ Our results suggest that the AF MSCs and WJ MSCs possess some characteristics of embryonic stem cells besides the characteristics of mesenchymal stromal cells.

Our data also showed both AF MSCs and WJ MSCs have myocardial-like cell differentiation capacity by real time PCR analysis. Two types of cells were respectively induced by 5 -aza-C. After induced differentiation in vitro, the AF MSCs and WJ MSCs were both positive for GATA-4, 
c-TnT, $\alpha$-actin and Cx43 mRNA. We also detected the corresponding protein expression by western blot analysis. GATA-4 is an important transcription factor in early myocardial development. c-TnT is a protein whose only known function is as part of the troponin complex of myofibrils in cardiac and embryonic skeletal muscles, ${ }^{[15]}$ while $\alpha$-actin is one of the isoforms of the actin family from muscle actin. ${ }^{[16]} \mathrm{Cx} 43$ is one of gap junction proteins, which is the dominant gap junction component in the atrial and ventricular myocytes. ${ }^{[17]}$ Therefore, our data revealed that the myocardial induced AF MSCs and WJ MSCs had myocardial-like cells characteristics, including the transcription factor in early myocardial development of GATA-4 and several mature myocardial genes c-TnT, $\alpha$-actin and Cx43.

In conclusion, our study demonstrates that AF MSCs and WJ MSCs had similar morphology and cells phenotypes. They also had similar myocardial-like cells differentiation capacity in vitro. However, the convenient harvesting method and relatively increased propagation rate of AF MSCs may suggest a potential better clinical use for regenerative therapy in heart failure after acute myocardial infarction.

\section{References}

1 Lloyd-Jones D, Adams RJ, Brown TM, et al. Executive summary: heart disease and stroke statistics-1010 update: a report from the American heart association. Circulation 2010; 121: 948-954.

2 Ma L, Feng XY, Cui BL, et al. Human umbilical cord Wharton's Jelly-derived mesenchymal stem cells differentiation into nervelike cells. Chin Med J 2005; 118: 1987-1993.

3 Chao KC, Chao KF, Fu YS, et al. Islet-Like Clusters Derived from Mesenchymal Stem Cells in Wharton's Jelly of the Human Umbilical Cord for Transplantation to Control Type 1 Diabetes. PLoS One 2008; 3: E1451.

4 Tsai PC, Fu TW, Chen YM, et al. The therapeutic potential of human umbilical mesenchymal stem cells from Wharton's Jelly in the treatment rat liver fibrosis. Liver Transpl 2009; 15 : 484-495.

5 Friedenstein AJ, Gorskaja JF, Kulagina NN. Fibroblast precursors in normal and irradiated mouse hematopoietic organs. Exp Hematol 1976; 4: 267-274.

6 Pittenger MF, Mackay AM, Beck SC, et al. Multilineage potential of adult human mesenchymal stem cells. Science 1999; 284: 143-147.

7 Lennon DP, Edmison JM, Caplan SI. Cutlivation of rat marrowderived mesenchymal stem cells in reduced oxygen tension: effects on in vitro and in vivo osteochondrogenesis. $J$ Cell Physiol 2001; 187: 345-355.

8 Sekiya I, Vuoristo JT, Larson BL, et al. In Vitro Cartilage formation by human adult stem cells from bone marrow stroma defines the sequence of cellular and molecular events during condrogenesis. Proc Natl Acad Sci USA 2002; 99: 4397-4402.

9 Prusa AR, Marton E, Rosner M, et al. Oct-4-expressing cells in human amniotic fluid: a new source for stem cell research? Hum Reprod 2003; 18: 1489-1493.

10 De Coppi P, BartschG Ir, Siddiqui MM, et al. Isolation of amniotic stem cell lines with potential for therapy. Nat Biotechnol 2007; 25: 100-106.

11 Sadler TW. Second week of development: Bilaminar germ disc. In Langmann's Medical Embryology; William \& Wilkins: Philadephia, USA, 2004; 51-64.

12 Liu LL, Liu YJ, Yang SG, et al. Isolation and characterization of human umbilicol cord mesenchymal stem cells with heamatoposesis-supportive function and other potential. Heamatologica 2006; 91: 1017-1026.

13 Wang HS, Hung SC, Peng ST, et al. Mesenchymal stem cells in Wharton's Jelly of the human umbilical cord. Stem Cells 2004; 22: 1330-1337.

14 Karahuseyinoglu S, Cinar O, Kilic E, et al. Biology of the stem cells of human umbilical cord stroma: in situ and in vitro surveys. Stem Cells 2007; 25: 319-331.

15 Parker BA, Mark AB, Zhang WJ, et al. Precocious expression of cardiac troponin $\mathrm{T}$ in early Chick embryos is independent of bone morphogenetic protein signaling. Dev Dyn 2002; 225: $135-141$.

16 Oma Y, Harata M. Actin-related proteins localized in the nucleus From discovery to novel roles in nuclear organization. Nucleus 2011; 2: 38-46.

17 Laird DW. The gap junction proteome and its relationship to disease. Trends Cell Biol 2010; 20: 92-101. 